\title{
Solutions of singular antiperiodic boundary value problems
}

\author{
Oto Přibyl
}




\title{
SOLUTIONS OF SINGULAR ANTIPERIODIC BOUNDARY VALUE PROBLEMS
}

\author{
OTO PŘIBYL
}

[Received: October 12, 2004]

\begin{abstract}
Sufficient conditions for the existence of a solution of the equation

$$
\left(g\left(x^{\prime}(t)\right)\right)^{\prime}=f\left(t, x(t), x^{\prime}(t)\right)
$$

with the antiperiodic conditions $x(0)+x(T)=0, x^{\prime}(0)+x^{\prime}(T)=0$ are established. Our nonlinearity $f$ may be singular at its phase variables. The proofs are based on a combination of regularity and sequential techniques and use the topological transversality principle.
\end{abstract}

Mathematics Subject Classification: 34B15, 34B16

Keywords: singular second-order differential equation, g-Laplacian, antiperiodic boundary conditions, topological transversality principle, Vitali's convergence theorem

\section{InTRODUCTION}

Let $T$ be a positive number, $J=[0, T], \mathbb{R}_{+}=(0, \infty)$ and $\mathbb{R}_{0}=\mathbb{R} \backslash\{0\}$. We will consider the differencial equation

$$
\left(g\left(x^{\prime}(t)\right)\right)^{\prime}=f\left(t, x(t), x^{\prime}(t)\right)
$$

together with the boundary conditions of the antiperiodic type

$$
x(0)+x(T)=0, \quad x^{\prime}(0)+x^{\prime}(T)=0 .
$$

Here $f$ satisfies the local Carathédory conditions on $J \times \mathbb{R}_{0}^{2}\left(f \in \operatorname{Car}\left(J \times \mathbb{R}_{0}^{2}\right)\right)$ and $f$ may be singular at the zero value of its phase variables.

A function $x \in C^{1}(J)$ is said to be a solution of the boundary value problem (BVP for short) (1.1), (1.2) if $g\left(x^{\prime}\right) \in A C(J)$ (that is $g\left(x^{\prime}\right)$ is absolutely continuous on $J$ ), $x$ satisfies the boundary conditions (1.2) and (1.1) is satisfied a. e. on $J$.

The object of the work is to give assumptions on the functions $g$ and $f$ in (1.1) which guarantee the solvability of BVP (1.1), (1.2). We recall that by our definition, solutions of BVP (1.1), (1.2) have the "maximal" smoothness (that is, $x \in C^{1}(J)$ and $\left.g\left(x^{\prime}\right) \in A C(J)\right)$. Also any solution and its derivative 'pass through' the singular points of $f$ at inner points of the interval $J$. Since our existence results are proved 
by regularization and sequential methods, we will use Vitali's convergence theorem (see [3]) in limiting processes since it is impossible to find an integrable majorant for applying the Lebesgue Dominated Convergence Theorem. Boundary conditions of the antiperiodic type appear in the literature usually as the special case of nonlocal boundary conditions. They were considered for regular (in phase variables) $n$-order differential equations (see, e. g., $[4,5]$ ), systems of differential equations (see, e. g., [8]) and second-order functional differential equations (see, e. g., [11]). In the literature there are only a few papers dealing with BVPs whose nonlinearities are singular at the phase variables and their solutinos and their derivatives 'pass through' singular points at inner points of the interval $J$. Such BVPs were discussed for higher order differential equations (see, e. g., $[1,9]$ ) and second-order differential equations (see, e. g., $[2,10,12-14])$. From now on, $\|x\|=\max \{|x(t)| ; t \in J\},\|x\|_{L}=\int_{0}^{T}|x(t)| d t$ and $\|x\|_{\infty}=\operatorname{ess} \sup \{|x(t)| ; t \in J\}$ stands for the norm in $C^{0}(J), L_{1}(J)$ and $L_{\infty}(J)$, respectively. For a measurable set $\mathcal{M} \subset \mathbb{R}, \mu(\mathcal{M})$ denotes the Lebesgue measure of $\mathcal{M}$. Throughout the paper, we assume that the following assumptions are satisfied:

$\left(H_{1}\right) g \in C^{0}(\mathbb{R})$ is an increasing and odd function on $\mathbb{R}$ and $\lim _{u \rightarrow \infty} g(u)=\infty$;

$\left(H_{2}\right) f \in \operatorname{Car}\left(J \times \mathbb{R}_{0}^{2}\right)$ and there exists $a \in \mathbb{R}_{+}$such that

$$
a \leq f(t, u, v) \text { for a. e. } t \in J \text { and all }(u, v) \in \mathbb{R}_{0}^{2} ;
$$

$\left(H_{3}\right)$ For a. e. $t \in J$ and all $(u, v) \in \mathbb{R}_{0}^{2}$,

$$
f(t, u, v) \leq h(|u|)\left[\omega_{1}(g(|v|))+\omega_{2}(g(|v|))\right]
$$

where $\omega_{1}:[0, \infty) \rightarrow \mathbb{R}_{+}$is continuous and increasing and

$$
\int_{0}^{\infty} \frac{1}{\omega_{1}(s)} d s=\infty
$$

$h, \omega_{2}: \mathbb{R}_{+} \rightarrow \mathbb{R}_{+}$are continuous and decreasing and

$$
\int_{0}^{1} h(s) d s<\infty, \quad \int_{0}^{1} \omega_{2}(s) d s<\infty . *
$$

The paper is organized as follows. Section 2 deals with a sequence of auxiliary regular BVPs to problem (1.1), (1.2). We give a priori bounds for their solutions $x_{n}$ and prove their existence by the topological transversality principle (see, e. g., [6] or [7]). In addition, we prove that the sequence $\left\{f_{n}\left(t, x_{n}(t), x_{n}^{\prime}(t)\right)\right\}$ is uniformly integrable on $J$. In Section 3, applying Vitali's convergence theorem, existence results to BVP (1.1), (1.2) are proved. We also include an example to illustrate our theory.

\footnotetext{
${ }^{*}$ Since $h, \omega_{2}: \mathbb{R}_{+} \rightarrow \mathbb{R}_{+}$are decreasing, the condition (1.4) implies that $\int_{0}^{V} h(s) d s<\infty$ and $\int_{0}^{V} \omega_{2}(s) d s<\infty$ for all $V>0$.
} 


\section{Auxiliary Regular BVPs AND their Properties}

For each $n \in \mathbb{N}$ and $\lambda \in[0,1]$, define $f_{n}$ and $h_{n}(\lambda ; \cdot) \in \operatorname{Car}\left(J \times \mathbb{R}^{2}\right)$ by

$$
f_{n}(t, u, v)=\left\{\begin{array}{c}
f(t, u, v) \text { for }(t, u, v) \in J \times\left(\mathbb{R} \backslash\left[-\frac{1}{n}, \frac{1}{n}\right]\right)^{2} ; \\
\frac{n}{2}\left[\left(u+\frac{1}{n}\right) \cdot f(t, 1 / n, v)-\left(u-\frac{1}{n}\right) \cdot f(t,-1 / n, v)\right] \\
\quad \text { for }(t, u, v) \in J \times\left[-\frac{1}{n}, \frac{1}{n}\right] \times\left(\mathbb{R} \backslash\left[-\frac{1}{n}, \frac{1}{n}\right]\right) ; \\
\frac{n}{2}\left[\left(v+\frac{1}{n}\right) \cdot f_{n}(t, u, 1 / n)-\left(v-\frac{1}{n}\right) \cdot f_{n}(t, u,-1 / n)\right] \\
\quad \text { for }(t, u, v) \in J \times \mathbb{R} \times\left[-\frac{1}{n}, \frac{1}{n}\right]
\end{array}\right.
$$

and

$$
h_{n}(\lambda ; t, u, v)=\lambda f_{n}(t, u, v)+(1-\lambda) a \quad \text { for }(t, u, v) \in J \times \mathbb{R}^{2} .
$$

Then

$$
a \leq h_{n}(\lambda ; t, u, v) \leq f_{n}(t, u, v) \text { for a. e. } t \in J \text { and each }(u, v) \in \mathbb{R}^{2}
$$

and

$$
f_{n}(t, u, v) \leq h(|u|)\left[\omega_{1}(g(|v|)+g(1))+\omega_{2}(g(|v|))\right]
$$

for a. e. $t \in J$ and each $(u, v) \in \mathbb{R}_{0}^{2}$.

Consider the family of regular differential equations

$$
\left(g\left(x^{\prime}(t)\right)\right)^{\prime}=h_{n}\left(\lambda ; t, x(t), x^{\prime}(t)\right)
$$

depending on the parameters $n \in \mathbb{N}$ and $\lambda \in[0,1]$.

Lemma 1. Let $x$ be a solution of $B V P$ (2.3), (1.2) with some $\lambda \in[0,1]$ and $n \in \mathbb{N}$ in (2.3). Then

(a) $x^{\prime}$ is increasing on $J$ and

$$
g\left(\left|x^{\prime}(t)\right|\right) \geq a|\xi-t| \quad \text { for } t \in[0, T]
$$

where $\xi \in(0, T)$ is the unique zero of $x^{\prime}$;

(b) $x$ has a unique zero $\eta$ on $[0, T), \eta \neq \xi$ and if $\xi<\eta$ then

$$
|x(t)| \geq \begin{cases}\frac{S}{\xi} t & \text { for } t \in[0, \xi] \\ \frac{S}{\eta-\xi}|t-\eta| & \text { for } t \in(\xi, T]\end{cases}
$$

and if $\eta<\xi$ then

$$
|x(t)| \geq \begin{cases}\frac{S}{\xi-\eta}|t-\eta| & \text { for } t \in[0, \xi] \\ \frac{S}{T-\xi}(T-t) & \text { for } t \in(\xi, T]\end{cases}
$$


where

$$
S=\frac{1}{2 a} \int_{0}^{\frac{a T}{2}} g^{-1}(s) d s .^{\dagger}
$$

Proof. Since $\left(g\left(x^{\prime}(t)\right)\right)^{\prime}=h_{n}\left(\lambda ; t, x(t), x^{\prime}(t)\right) \geq a$ for a. e. $t \in J$ by (2.1) and $g$ is increasing on $\mathbb{R}$, we see that $x^{\prime}$ is increasing on $J$ and from $x^{\prime}(0)=-x^{\prime}(T)$ it follows that $x^{\prime}$ has a unique zero $\xi \in(0, T)$ and then $x^{\prime}<0$ on $[0, \xi)$ and $x^{\prime}>0$ on $(\xi, T]$. Next, we have

$$
\begin{gathered}
-g\left(x^{\prime}(t)\right)=\int_{t}^{\xi}\left(g\left(x^{\prime}(s)\right)\right)^{\prime} d s \geq a(\xi-t), \quad t \in[0, \xi], \\
g\left(x^{\prime}(t)\right)=\int_{\xi}^{t}\left(g\left(x^{\prime}(s)\right)\right)^{\prime} d s \geq a(t-\xi), \quad t \in(\xi, T]
\end{gathered}
$$

which gives (2.4). Since $x$ is decreasing on $[0, \xi]$ and increasing on $[\xi, T]$, we see that if $x(0)<0$ (resp. $x(0)>0)$, then $x(T)=-x(0)>0($ resp. $x(T)<0)$, and so $x$ vanishes at a unique $\eta \in(\xi, T)$ (resp. $\eta \in(0, \xi)$ ). In the case of $x(0)=0$, we have $x(T)=0$ and $x<0$ on $(0, T)$. We have proved that $x$ has a unique zero $\eta$ in the interval $[0, T)$. Now from (2.4) we get

$$
\begin{aligned}
& x(T)-x(\xi)=\int_{\xi}^{T} x^{\prime}(t) d t \geq \int_{\xi}^{T} g^{-1}(a(t-\xi)) d t=\frac{1}{a} \int_{0}^{a(T-\xi)} g^{-1}(s) d s, \\
& x(\xi)-x(0)=\int_{0}^{\xi} x^{\prime}(t) d t \leq-\int_{0}^{\xi} g^{-1}(a(\xi-t)) d t=-\frac{1}{a} \int_{0}^{a \xi} g^{-1}(s) d s,
\end{aligned}
$$

and so

$$
-x(\xi)-\frac{1}{a} \int_{0}^{a \xi} g^{-1}(s) d s \geq-x(0)=x(T) \geq x(\xi)+\frac{1}{a} \int_{0}^{a(T-\xi)} g^{-1}(s) d s .
$$

We get

$$
\begin{aligned}
x(\xi) & \leq-\frac{1}{2 a}\left[\int_{0}^{a \xi} g^{-1}(s) d s+\int_{0}^{a(T-\xi)} g^{-1}(s) d s\right] \\
& \leq-\frac{1}{2 a} \int_{0}^{a \max \{\xi, T-\xi\}} g^{-1}(s) d s .
\end{aligned}
$$

Since $\max \{\xi, T-\xi\} \geq T / 2$, we have $|x(\xi)| \geq S$ where $S$ is defined by (2.7). (2.5) for $\xi<\eta$ or (2.6) for $\eta<\xi$ now follows from the convexity of $x$ on $J$.

Lemma 2. Let $x$ be a solution of $B V P(2.3)$, (1.2) with some $\lambda \in[0,1]$ and $n \in \mathbb{N}$ in (2.3). Then there exists a positive constant $P$ independent of $\lambda$ and $n$ such that

$$
\left\|x^{\prime}\right\|<P \text {. }
$$

\footnotetext{
${ }^{\dagger}$ Here and subsequently, $g^{-1}: \mathbb{R} \rightarrow \mathbb{R}$ denotes the inverse function to $g$.
} 
Proof. By Lemma 1, $x^{\prime}$ is increasing on $J$ and has a unique zero $\xi \in(0, T)$. Hence

$$
\left\|x^{\prime}\right\|=\max \left\{-x^{\prime}(0), x^{\prime}(T)\right\} \text {. }
$$

We are going to give upper bounds for $-x^{\prime}(0)$ and $x^{\prime}(T)$. Since

$$
\left(g\left(x^{\prime}(t)\right)\right)^{\prime}=h_{n}\left(\lambda ; t, x(t), x^{\prime}(t)\right) \leq f_{n}\left(t, x(t), x^{\prime}(t)\right)
$$

for a. e. $t \in J$ by (2.1), (2.2) shows that

$$
\frac{\left(g\left(x^{\prime}(t)\right)\right)^{\prime}}{\omega_{1}\left(-g\left(x^{\prime}(t)\right)+g(1)\right)+\omega_{2}\left(-g\left(x^{\prime}(t)\right)\right)} \leq h(|x(t)|) \quad \text { for a. e. } t \in[0, \xi]
$$

and

$$
\frac{\left(g\left(x^{\prime}(t)\right)\right)^{\prime}}{\omega_{1}\left(g\left(x^{\prime}(t)\right)+g(1)\right)+\omega_{2}\left(g\left(x^{\prime}(t)\right)\right)} \leq h(|x(t)|) \quad \text { for a. e. } t \in[\xi, T]
$$

because of $x^{\prime}<0$ on $[0, \xi), x^{\prime}(t)>0$ on $(\xi, T]$ and $g$ is an odd function by $\left(H_{1}\right)$. To find upper estimates for $\int_{0}^{\xi} h(|x(t)|) d t$ and $\int_{\xi}^{T} h(|x(t)|) d t$ we must distinguish two cases according to the values of $\xi$ and $\eta$ :

Case 1. If $\xi<\eta$, then by (2.5)

$$
\int_{0}^{\xi} h(|x(t)|) d t \leq \int_{0}^{\xi} h\left(\frac{S}{\xi} t\right) d t \leq \frac{T}{S} \int_{0}^{S} h(s) d s
$$

and

$$
\begin{aligned}
\int_{\xi}^{T} h(|x(t)|) d t & \leq \int_{\xi}^{T} h\left(\frac{S}{\eta-\xi}|t-\eta|\right) d t \\
& \leq \int_{\xi}^{\eta} h\left(\frac{S}{\eta-\xi}(\eta-t)\right) d t+\int_{\eta}^{T} h\left(\frac{S}{T}(t-\eta)\right) d t \\
& \leq \frac{\eta-\xi}{S} \int_{0}^{S} h(s) d s+\frac{T}{S} \int_{0}^{S \frac{T-\eta}{T}} h(s) d s \\
& \leq \frac{2 T}{S} \int_{0}^{S} h(s) d s .
\end{aligned}
$$

Case 2. If $\eta<\xi$, then by (2.6)

$$
\begin{aligned}
\int_{0}^{\xi} h(|x(t)|) d t & \leq \int_{0}^{\xi} h\left(\frac{S}{\xi-\eta}|t-\eta|\right) d t \\
& \leq \int_{0}^{\eta} h\left(\frac{S}{T}(\eta-t)\right) d t+\int_{\eta}^{\xi} h\left(\frac{S}{\xi-\eta}(t-\eta)\right) d t \\
& \leq \frac{T}{S} \int_{0}^{S \frac{\eta}{T}} h(s) d s+\frac{\xi-\eta}{S} \int_{0}^{S} h(s) d s \\
& \leq \frac{2 T}{S} \int_{0}^{S} h(s) d s
\end{aligned}
$$


and

$$
\int_{\xi}^{T} h(|x(t)|) d t \leq \int_{\xi}^{T} h\left(\frac{S}{T-\xi}(T-t)\right) d t \leq \frac{T}{S} \int_{0}^{S} h(s) d s
$$

Let

Since

$$
Q=1+\frac{2 T}{S} \int_{0}^{S} h(s) d s .
$$

$$
\begin{aligned}
& \int_{0}^{\xi} \frac{\left(g\left(x^{\prime}(t)\right)\right)^{\prime}}{\omega_{1}\left(-g\left(x^{\prime}(t)\right)+g(1)\right)+\omega_{2}\left(-g\left(x^{\prime}(t)\right)\right)} d t \\
& \quad=\int_{0}^{-g\left(x^{\prime}(0)\right)} \frac{1}{\omega_{1}(s+g(1))+\omega_{2}(s)} d s
\end{aligned}
$$

and

$$
\begin{aligned}
\int_{\xi}^{T} \frac{\left(g\left(x^{\prime}(t)\right)\right)^{\prime}}{\omega_{1}\left(g\left(x^{\prime}(t)\right)+g(1)\right)+\omega_{2}\left(g\left(x^{\prime}(t)\right)\right)} d t & \\
& =\int_{0}^{g\left(x^{\prime}(T)\right)} \frac{1}{\omega_{1}(s+g(1))+\omega_{2}(s)} d s,
\end{aligned}
$$

integrating (2.10) over $[0, \xi]$ and using (2.16) we get

$$
\int_{0}^{-g\left(x^{\prime}(0)\right)} \frac{1}{\omega_{1}(s+g(1))+\omega_{2}(s)} d s<Q
$$

and integrating (2.11) over $[\xi, T]$ and using (2.17) we get

$$
\int_{0}^{g\left(x^{\prime}(T)\right)} \frac{1}{\omega_{1}(s+g(1))+\omega_{2}(s)} d s<Q .
$$

Since $\int_{0}^{\infty} \frac{1}{\omega_{1}(s)} d s=\infty$ by (1.3), we see that $\int_{0}^{\infty} \frac{1}{\omega_{1}(s+g(1))} d s=\infty$ and then

$$
\int_{0}^{\infty} \frac{1}{\omega_{1}(s+g(1))+\omega_{2}(s)} d s=\infty
$$

as well. Therefore there exists $P_{1} \in \mathbb{R}_{+}$such that

$$
\int_{0}^{u} \frac{1}{\omega_{1}(s+g(1))+\omega_{2}(s)} d s>Q \quad \text { for } u \geq P_{1} .
$$

Now from the last inequality, (2.18) and (2.19) we deduce that

$$
\max \left\{-g\left(x^{\prime}(0)\right), g\left(x^{\prime}(T)\right)\right\}<P_{1} .
$$

Hence $\max \left\{-x^{\prime}(0), x^{\prime}(T)\right\}<g^{-1}\left(P_{1}\right)$ and then (2.9) shows that is true (2.8) with $P=$ $g^{-1}\left(P_{1}\right)$.

The lemma is proved.

Lemma 3. For each $n \in \mathbb{N}$ there exists a solution of $B V P(2.3),(1.2)$ with $\lambda=1$ in (2.3). 
Proof. Fix $n \in \mathbb{N}$ and let $P$ be a positive constant given in Lemma 2. Set

$$
\Omega=\left\{x \in C^{1}(J) ;\|x\|<R T,\left\|x^{\prime}\right\|<R\right\}
$$

where $R=\max \left\{P, g^{-1}(a T)\right\}$. Then $\Omega$ is an open, bounded and convex subset of the Banach space $C^{1}(J)$. Define the operators

$$
\Pi:[0,1] \times \bar{\Omega} \rightarrow C^{0}(J) \quad \text { and } \quad \mathcal{K}:[0,1] \times \bar{\Omega} \rightarrow C^{1}(J)
$$

by the formulas

$$
\Pi(\lambda, x)(t)=g^{-1}\left(\frac{1}{2} \int_{0}^{t} h_{n}\left(\lambda ; s, x(s), x^{\prime}(s)\right) d s-\frac{1}{2} \int_{t}^{T} h_{n}\left(\lambda ; s, x(s), x^{\prime}(s)\right) d s\right)
$$

and

$$
\mathcal{K}(\lambda, x)(t)=\frac{1}{2} \int_{0}^{t} \Pi(\lambda, x)(s) d s-\frac{1}{2} \int_{t}^{T} \Pi(\lambda, x)(s) d s .
$$

It is easy to check that $x$ is a solution of BVP (2.3), (1.2) with $\lambda=1$ in (2.3) if and only if $x$ is a fixed point of the operator $\mathcal{K}(1, \cdot)$. We now show that $\mathcal{K}(1, \cdot)$ has a fixed point. By the topological transversality principle, it is sufficient to verify that:

(i) $\mathcal{K}$ is a compact operator,

(ii) $\mathcal{K}(\lambda, x) \neq x$ for each $(\lambda, x) \in[0,1] \times \partial \Omega$ and

(iii) $\mathcal{K}(0, \cdot)$ is a constant operator and $\mathcal{K}(0, x)=\hat{x}$ for $x \in \bar{\Omega}$ with an $\hat{x} \in \Omega$.

Since $h_{n}(\lambda ; \cdot) \in \operatorname{Car}\left(J \times \mathbb{R}^{2}\right)$, there exists $q \in L_{1}(J)$ such that

$$
\left|h_{n}(\lambda ; t, u, v)\right| \leq q(t)
$$

for a. e. $t \in J$ and each $\lambda \in[0,1], u \in[-R T, R T], v \in[-R, R]$.

Let $\left\{\lambda_{k}\right\} \subset[0,1],\left\{x_{k}\right\} \subset \bar{\Omega}$ be convergent sequences such that $\lim _{k \rightarrow \infty} \lambda_{k}=\lambda_{*}$ and $\lim _{k \rightarrow \infty} x_{k}=x_{*}$. Since

$$
\begin{aligned}
& \left|g\left(\Pi\left(\lambda_{k}, x_{k}\right)(t)\right)-g\left(\Pi\left(\lambda_{*}, x_{*}\right)(t)\right)\right| \\
& \quad \leq \int_{0}^{T}\left|h_{n}\left(\lambda_{k} ; s, x_{k}(s), x_{k}^{\prime}(s)\right)-h_{n}\left(\lambda_{*} ; s, x_{*}(s), x_{*}^{\prime}(s)\right)\right| d s,
\end{aligned}
$$

using the Lebesgue dominated convergence theorem we see that

$$
\lim _{k \rightarrow \infty}\left\|g\left(\Pi\left(\lambda_{k}, x_{k}\right)\right)-g\left(\Pi\left(\lambda_{*}, x_{*}\right)\right)\right\|=0 .
$$

Then from the continuity of $g^{-1}$ on $\mathbb{R}$ it follows that

$$
\lim _{k \rightarrow \infty}\left\|\Pi\left(\lambda_{k}, x_{k}\right)-\Pi\left(\lambda_{*}, x_{*}\right)\right\|=\lim _{k \rightarrow \infty}\left\|g^{-1}\left(g\left(\Pi\left(\lambda_{k}, x_{k}\right)\right)\right)-g^{-1}\left(g\left(\Pi\left(\lambda_{*}, x_{*}\right)\right)\right)\right\|=0 .
$$

Now from the inequalities

$$
\left|\mathcal{K}\left(\lambda_{k}, x_{k}\right)(t)-\mathcal{K}\left(\lambda_{*}, x_{*}\right)(t)\right| \leq \int_{0}^{T}\left|\Pi\left(\lambda_{k}, x_{k}\right)(s)-\Pi\left(\lambda_{*}, x_{*}\right)(s)\right| d s
$$


and

$$
\begin{aligned}
\left|\left(\mathcal{K}\left(\lambda_{k}, x_{k}\right)\right)^{\prime}(t)-\left(\mathcal{K}\left(\lambda_{*}, x_{*}\right)\right)^{\prime}(t)\right| & =\left|\Pi\left(\lambda_{k}, x_{k}\right)(t)-\Pi\left(\lambda_{*}, x_{*}\right)(t)\right| \\
& \leq\left\|\Pi\left(\lambda_{k}, x_{k}\right)-\Pi\left(\lambda_{*}, x_{*}\right)(s)\right\|,
\end{aligned}
$$

we get $\lim _{k \rightarrow \infty} \mathcal{K}\left(\lambda_{k}, x_{k}\right)=\mathcal{K}\left(\lambda_{*}, x_{*}\right)$ in $C^{1}(J)$, which proves that $\mathcal{K}$ is a continuous operator.

Let now $\left\{\left(\bar{\lambda}_{k}, \bar{x}_{k}\right)\right\} \subset[0,1] \times \bar{\Omega}$ and consider the sequence $\left\{\mathcal{K}\left(\bar{\lambda}_{k}, \bar{x}_{k}\right)\right\}$. Then (see (2.20))

$$
\left|\mathcal{K}\left(\bar{\lambda}_{k}, \bar{x}_{k}\right)(t)\right| \leq T g^{-1}\left(\|q\|_{L}\right), \quad\left|\left(\mathcal{K}\left(\bar{\lambda}_{k}, \bar{x}_{k}\right)\right)^{\prime}(t)\right| \leq g^{-1}\left(\|q\|_{L}\right),
$$

and

$$
\left|g\left[\left(\mathcal{K}\left(\bar{\lambda}_{k}, \bar{x}_{k}\right)\right)^{\prime}\left(t_{1}\right)\right]-g\left[\left(\mathcal{K}\left(\bar{\lambda}_{k}, \bar{x}_{k}\right)\right)^{\prime}\left(t_{2}\right)\right]\right| \leq \int_{t_{1}}^{t_{2}} q(t) d t
$$

for $k \in \mathbb{N}, t \in J$ and $0 \leq t_{2} \leq t_{1} \leq T$. Therefore $\left\{\mathcal{K}\left(\bar{\lambda}_{k}, \bar{x}_{k}\right)\right\}$ is bounded in $C^{1}(J)$ and from (2.21) we deduce that $\left\{\left(\mathcal{K}\left(\bar{\lambda}_{k}, \bar{x}_{k}\right)\right)^{\prime}(t)\right\}$ is equicontinuous on J. By the ArzelàAscoli theorem, there is a convergent subsequence of $\left\{\mathcal{K}\left(\bar{\lambda}_{k}, \bar{x}_{k}\right)\right\}$ in $C^{1}(J)$. Hence $\mathcal{K}([0,1] \times \bar{\Omega})$ is relatively compact and since $\mathcal{K}$ is continuous, that is a compact operator.

Assume that $\mathcal{K}\left(\lambda_{0}, x_{0}\right)=x_{0}$ for some $\left(\lambda_{0}, x_{0}\right) \in[0,1] \times \partial \Omega$. Then $x_{0}$ is a solution of BVP (2.3), (1.2) with $\lambda=\lambda_{0}$ in (2.3), and so $\left\|x_{0}^{\prime}\right\|<P$ by Lemma 2. From $x_{0}(0)=-x_{0}(T)$ it follows that $x_{0}$ has a zero $\eta \in J$. Now

$$
\left|x_{0}(t)\right|=\left|x_{0}(t)-x_{0}(\eta)\right| \leq\left\|x_{0}^{\prime}\right\||t-\eta|
$$

yields $\left\|x_{0}\right\|<P T$, contrary to $x_{0} \in \partial \Omega$. Hence $\mathcal{K}(\lambda, x) \neq x$ for all $(\lambda, x) \in[0,1] \times \partial \Omega$.

Finally, for $x \in \bar{\Omega}$ and $t \in J$

$$
\mathcal{K}(0, x)(t)=\frac{1}{2} \int_{0}^{t} g^{-1}(a(s-T / 2)) d s-\frac{1}{2} \int_{t}^{T} g^{-1}(a(s-T / 2)) d s .
$$

Since $g^{-1}$ is increasing and odd on $\mathbb{R}$, we get

$$
\left|(\mathcal{K}(0, x))^{\prime}(t)\right|=\left|g^{-1}(a(t-T / 2))\right|=g^{-1}(a|t-T / 2|) \leq g^{-1}(a T / 2)
$$

for $t \in J$. Hence (iii) holds with $\hat{x}(t) \in \Omega$ defined by the righthand side of equality (2.22).

Lemma 4. For each $n \in \mathbb{N}$, let $x_{n}$ be a solution of $B V P(2.3),(1.2)$ with $\lambda=1$ in (2.3) and let $\eta_{n}$ be the unique zero of $x_{n}$ lying in $[0, T)$ and $\xi_{n}$ be the unique zero of $x_{n}^{\prime}$. Then there exists a positive constant $\Delta$ independent of $n$ such that

$$
\Delta \leq \frac{\left|\xi_{n}-\eta_{n}\right|}{2} \quad \text { for } n \in \mathbb{N} .
$$

Proof. Applying Lemmas 1 and 2, there exist positive constants $S$ and $P$ such that

$$
\left|x_{n}\left(\xi_{n}\right)\right| \geq S, \quad\left\|x_{n}^{\prime}\right\|<P, \quad n \in \mathbb{N} .
$$


Next (for $n \in \mathbb{N})$

$$
S \leq\left|x_{n}\left(\xi_{n}\right)\right|=\left|x_{n}\left(\xi_{n}\right)-x_{n}\left(\eta_{n}\right)\right| \leq\left\|x_{n}^{\prime}\right\|\left|\xi_{n}-\eta_{n}\right|<P\left|\xi_{n}-\eta_{n}\right| .
$$

Consequently (2.23) holds with $\Delta=\frac{S}{2 P}$.

Lemma 5. For each $n \in \mathbb{N}$, let $x_{n}$ be a solution of $B V P(2.3),(1.2)$ with $\lambda=1$ in (2.3) and let $\xi_{n}$ be the unique zero of $x_{n}^{\prime}$. Then for any subsequence $\left\{x_{k_{n}}\right\}$ of $\left\{x_{n}\right\}$ satisfying

$$
\left|x_{k_{n}}(T)\right| \leq \frac{S}{T+1} \quad \text { for } n \in \mathbb{N}
$$

with $S$ given by (2.7), there exists its subsequence $\left\{x_{l_{n}}\right\}$ and a positive constant $c$ independent of $n$ such that

$$
c \leq \xi_{l n} \leq T-c \quad \text { for } n \in \mathbb{N} .
$$

Proof. By Lemma $1,\left|x_{n}\left(\xi_{n}\right)\right| \geq S$ for $n \in \mathbb{N}$ and Lemma 2 guarantees the existence of a positive constant $P$ such that $\left\|x_{n}^{\prime}\right\|<P$ for $n \in \mathbb{N}$. Let $\left\{x_{k_{n}}\right\} \subset\left\{x_{n}\right\}$ be an arbitrary subsequence satisfying (2.24). Consider the sequences

$$
\left\{x_{k_{n}}\left(\xi_{k_{n}}\right)\right\},\left\{x_{k_{n}}(T)\right\} \subset[-P T, P T] .
$$

On account of the Bolzano-Weierstrass theorem, we see that there exists a subsequence $\left\{l_{n}\right\}$ of $\left\{k_{n}\right\}$ such that $\left\{x_{l_{n}}\left(\xi_{l_{n}}\right)\right\}$ and $\left\{x_{l_{n}}(T)\right\}$ are convergent in $\mathbb{R}$. Let

$$
\lim _{n \rightarrow \infty} x_{l_{n}}\left(\xi_{l_{n}}\right)=\alpha, \quad \lim _{n \rightarrow \infty} x_{l_{n}}(T)=\beta .
$$

Naturally,

Also

$$
|\beta| \leq \frac{S}{T+1}<|\alpha|
$$

and

$$
\left|x_{l_{n}}(T)-x_{l_{n}}\left(\xi_{l_{n}}\right)\right|<P\left(T-\xi_{l_{n}}\right)
$$

$$
\left|x_{l_{n}}(T)+x_{l_{n}}\left(\xi_{l_{n}}\right)\right|=\left|x_{l_{n}}\left(\xi_{l_{n}}\right)-x_{l_{n}}(0)\right|<P \xi_{l_{n}} .
$$

Assume that $\sup \left\{\xi_{l_{n}} ; n \in \mathbb{N}\right\}=T$ (resp. $\inf \left\{\xi_{l_{n}} ; n \in \mathbb{N}\right\}=0$ ). Then (2.28) (resp. (2.29)) gives $\beta-\alpha=0$ (resp., $\beta+\alpha=0$ ). Therefore

$$
|\alpha|=|\beta|
$$

contrary to (2.27). Hence there exists a positive constant $c$ such that relation (2.25) is satisfied.

Lemma 6. For each $n \in \mathbb{N}$, let $x_{n}$ be a solution of $B V P(2.3),(1.2)$ with $\lambda=1$ in (2.3). Then there exists a subsequence $\left\{x_{k_{n}}\right\} \subset\left\{x_{n}\right\}$ such that the sequence

$$
\left\{f_{k_{n}}\left(t, x_{k_{n}}(t), x_{k_{n}}^{\prime}(t)\right)\right\} \subset L_{1}(J)
$$

is uniformly integrable (UI) on J, that is for each $\varepsilon>0$ there exists $\delta>0$ such that

$$
\int_{\mathcal{M}}\left|f_{k_{n}}\left(t, x_{k_{n}}(t), x_{k_{n}}^{\prime}(t)\right)\right| d t<\varepsilon, \quad n \in \mathbb{N}
$$


whenever $\mathcal{M} \subset J$ is a measurable set and $\mu(\mathcal{M})<\delta$.

Proof. For $n \in \mathbb{N}$, let $\eta_{n} \in[0, T)$ and $\xi_{n} \in(0, T)$ be the unique zero of $x_{n}$ on $[0, T)$ and $x_{n}^{\prime}$, respectively. Let

$$
\Lambda=\min \left\{a, \frac{S}{T+1}\right\}
$$

where $a$ appears in $\left(H_{2}\right)$ and $S$ is given by (2.7). By Lemmas 2 and 4, there exist positive constants $P$ and $\Delta$ such that

$$
\left\|x_{n}^{\prime}\right\|<P, \quad 2 \Delta \leq\left|\xi_{n}-\eta_{n}\right| \quad \text { for } n \in \mathbb{N} .
$$

In addition (2.1), (2.2) and (2.32) give

$$
a \leq f_{n}\left(t, x_{n}(t), x_{n}^{\prime}(t)\right) \leq h\left(\left|x_{n}(t)\right|\right)\left[\omega_{1}(g(P)+g(1))+\omega_{2}\left(g\left(\left|x_{n}^{\prime}(t)\right|\right)\right)\right]
$$

for a. e. $t \in J$ and each $n \in \mathbb{N}$. Since

$$
h\left(\left|x_{n}(t)\right|\right) \leq \frac{1}{\omega_{2}(g(P))} h\left(\left|x_{n}(t)\right|\right) \omega_{2}\left(g\left(\left|x_{n}^{\prime}(t)\right|\right)\right)
$$

for a. e. $t \in J$ and any $n \in \mathbb{N}$, we see that to prove our lemma it suffices to show that there exists a subsequence $\left\{x_{k_{n}}\right\} \subset\left\{x_{n}\right\}$ such that the sequence

$$
\left\{h\left(\left|x_{k_{n}}(t)\right|\right) \omega_{2}\left(g\left(\left|x_{k_{n}}^{\prime}(t)\right|\right)\right)\right\}
$$

is UI on $J$.

From the structure of the measurable sets in $\mathbb{R}$ we deduce that the sequence (2.33) is UI on $J$ if for each $\varepsilon>0$ there exists $\delta>0$ such that for any at most countable sequence $\left\{\left(a_{j}, b_{j}\right)\right\}_{j \in \mathrm{J}}$ of mutually disjoint intervals $\left(a_{j}, b_{j}\right) \subset J$ such that $\sum_{j \in \mathbb{J}}\left(b_{j}-\right.$ $\left.a_{j}\right)<\delta$, we have

$$
\sum_{j \in \mathbb{J}} \int_{a_{j}}^{b_{j}} h\left(\left|x_{k_{n}}(t)\right|\right) \omega_{2}\left(g\left(\left|x_{k_{n}}^{\prime}(t)\right|\right)\right) d t<\varepsilon, \quad n \in \mathbb{N} .
$$

For this, let $\left\{\left(a_{j}, b_{j}\right)\right\}_{j \in \mathbb{J}}$ be an at most countable sequence of mutually disjoint intervals $\left(a_{j}, b_{j}\right) \subset J$ and assume that

$$
b_{j}-a_{j} \leq \Delta / 2 \quad \text { for each } j \in \mathbb{J} .
$$

In addition, by (2.32), we have

$$
\frac{\left|\xi_{n}-\eta_{n}\right|}{2} \geq \Delta>\frac{\Delta}{2} \geq b_{j}-a_{j} \quad \text { for } n \in \mathbb{N}, j \in \mathbb{J} .
$$

It is easy to see that there exists a subsequence $\left\{x_{k_{n}}\right\} \subset\left\{x_{n}\right\}$ such that one of the following two cases may occur, namely, Case 1 where $\xi_{k_{n}}>\eta_{k_{n}}$ for $n \in \mathbb{N}$, and Case 2 where $\xi_{k_{n}}<\eta_{k_{n}}$ for $n \in \mathbb{N}$. 
Case 1. Let $\xi_{k_{n}}>\eta_{k_{n}}$ for $n \in \mathbb{N}$. Since (see (2.32))

$$
\xi_{k_{n}}-t \geq \xi_{k_{n}}-\frac{\xi_{k_{n}}+\eta_{k_{n}}}{2} \geq \frac{\xi_{k_{n}}-\eta_{k_{n}}}{2} \geq \Delta, \quad t \in\left[0,\left(\xi_{k_{n}}+\eta_{k_{n}}\right) / 2\right]
$$

and

$$
t-\eta_{k_{n}} \geq \frac{\xi_{k_{n}}+\eta_{k_{n}}}{2}-\eta_{k_{n}} \geq \frac{\xi_{k_{n}}-\eta_{k_{n}}}{2} \geq \Delta, \quad t \in\left[\left(\xi_{k_{n}}+\eta_{k_{n}}\right) / 2, \xi_{k_{n}}\right],
$$

we have (by Lemma 1)

$$
g\left(\left|x_{k_{n}}^{\prime}(t)\right|\right) \geq \begin{cases}\Lambda \Delta & \text { for } t \in\left[0,\left(\eta_{k_{n}}+\xi_{k_{n}}\right) / 2\right], \\ \Lambda\left|t-\xi_{k_{n}}\right| & \text { for } t \in\left[\left(\eta_{k_{n}}+\xi_{k_{n}}\right) / 2, T\right]\end{cases}
$$

and

$$
\left|x_{k_{n}}(t)\right| \geq \begin{cases}\Lambda\left|t-\eta_{k_{n}}\right| & \text { for } t \in\left[0,\left(\eta_{k_{n}}+\xi_{k_{n}}\right) / 2\right], \\ \Lambda \Delta & \text { for } t \in\left[\left(\eta_{k_{n}}+\xi_{k_{n}}\right) / 2, \xi_{k_{n}}\right], \\ \Lambda(T-t) & \text { for } t \in\left[\xi_{k_{n}}, T\right]\end{cases}
$$

where $\Lambda$ is given by (2.31).

For $n \in \mathbb{N}$, set

$$
\mathbb{K}_{k_{n}}=\left\{j \in \mathbb{J} ; b_{j} \leq \xi_{k_{n}}\right\}, \quad \mathbb{L}_{k_{n}}=\mathbb{J} \backslash \mathbb{K}_{k_{n}}
$$

If $j \in \mathbb{K}_{k_{n}}$ and (see (2.35))

(a) $b_{j} \leq \eta_{k_{n}}$, then (see (2.36) and (2.37))

$$
\begin{aligned}
\int_{a_{j}}^{b_{j}} h\left(\left|x_{k_{n}}(t)\right|\right) \omega_{2}\left(g\left(\left|x_{k_{n}}^{\prime}(t)\right|\right)\right) d t & \leq \omega_{2}(\Lambda \Delta) \int_{a_{j}}^{b_{j}} h\left(\Lambda\left(\eta_{k_{n}}-t\right)\right) d t \\
& =\frac{\omega_{2}(\Lambda \Delta)}{\Lambda} \int_{\Lambda\left(\eta_{k_{n}}-b_{j}\right)}^{\Lambda\left(\eta_{k_{n}}-a_{j}\right)} h(s) d s
\end{aligned}
$$

(b) $a_{j} \leq \eta_{k_{n}}<b_{j}<\left(\eta_{k_{n}}+\xi_{k_{n}}\right) / 2$, then

$$
\begin{aligned}
\int_{a_{j}}^{b_{j}} h\left(\left|x_{k_{n}}(t)\right|\right) \omega_{2}\left(g\left(\left|x_{k_{n}}^{\prime}(t)\right|\right)\right) d t \\
\quad \leq \omega_{2}(\Lambda \Delta)\left(\int_{a_{j}}^{\eta_{k_{n}}} h\left(\Lambda\left(\eta_{k_{n}}-t\right)\right) d t+\int_{\eta_{k_{n}}}^{b_{j}} h\left(\Lambda\left(t-\eta_{k_{n}}\right)\right) d t\right) \\
\quad=\frac{\omega_{2}(\Lambda \Delta)}{\Lambda}\left(\int_{0}^{\Lambda\left(\eta_{k_{n}}-a_{j}\right)} h(s) d s+\int_{0}^{\Lambda\left(b_{j}-\eta_{k_{n}}\right)} h(s) d s\right)
\end{aligned}
$$


(c) $\eta_{k_{n}}<a_{j}<b_{j} \leq\left(\eta_{k_{n}}+\xi_{k_{n}}\right) / 2$, then

$$
\begin{aligned}
\int_{a_{j}}^{b_{j}} h\left(\left|x_{k_{n}}(t)\right|\right) \omega_{2}\left(g\left(\left|x_{k_{n}}^{\prime}(t)\right|\right)\right) d t & \leq \omega_{2}(\Lambda \Delta) \int_{a_{j}}^{b_{j}} h\left(\Lambda\left(t-\eta_{k_{n}}\right)\right) d t \\
& =\frac{\omega_{2}(\Lambda \Delta)}{\Lambda} \int_{\Lambda\left(a_{j}-\eta_{k_{n}}\right)}^{\Lambda\left(b_{j}-\eta_{k_{n}}\right)} h(s) d s
\end{aligned}
$$

(d) $\eta_{k_{n}}<a_{j}<\left(\eta_{k_{n}}+\xi_{k_{n}}\right) / 2<b_{j}$, then

$$
\begin{aligned}
\int_{a_{j}}^{b_{j}} h\left(\left|x_{k_{n}}(t)\right|\right) \omega_{2}\left(g\left(\left|x_{k_{n}}^{\prime}(t)\right|\right)\right) d t & \\
\leq & \omega_{2}(\Lambda \Delta) \int_{a_{j}}^{\left(\eta_{k_{n}}+\xi_{k_{n}}\right) / 2} h\left(\Lambda\left(t-\eta_{k_{n}}\right)\right) d t \\
& \quad+h(\Lambda \Delta) \int_{\left(\eta_{k_{n}}+\xi_{k_{n}}\right) / 2}^{b_{j}} \omega_{2}\left(\Lambda\left(\xi_{k_{n}}-t\right)\right) d t \\
\quad & \frac{\omega_{2}(\Lambda \Delta)}{\Lambda} \int_{\Lambda\left(a_{j}-\eta_{k_{n}}\right)}^{\Lambda\left(\xi_{k_{n}}-\eta_{k_{n}}\right) / 2} h(s) d s+\frac{h(\Lambda \Delta)}{\Lambda} \int_{\Lambda\left(\xi_{k_{n}}-b_{j}\right)}^{\Lambda\left(\xi_{k_{n}}-\eta_{k_{n}}\right) / 2} \omega_{2}(s) d s ;
\end{aligned}
$$

(e) $\left(\eta_{k_{n}}+\xi_{k_{n}}\right) / 2 \leq a_{j}$, then (see (2.38))

$$
\begin{aligned}
\int_{a_{j}}^{b_{j}} h\left(\left|x_{k_{n}}(t)\right|\right) \omega_{2}\left(g\left(\left|x_{k_{n}}^{\prime}(t)\right|\right)\right) d t & \leq h(\Lambda \Delta) \int_{a_{j}}^{b_{j}} \omega_{2}\left(\Lambda\left(\xi_{k_{n}}-t\right)\right) d t \\
& =\frac{h(\Lambda \Delta)}{\Lambda} \int_{\Lambda\left(\xi_{k_{n}}-b_{j}\right)}^{\Lambda\left(\xi_{k_{n}}-a_{j}\right)} \omega_{2}(s) d s .
\end{aligned}
$$

Summarizing, we have proved that

$$
\begin{aligned}
\sum_{j \in \mathbb{K}_{k_{n}}} \int_{a_{j}}^{b_{j}} h\left(\left|x_{k_{n}}(t)\right|\right) & \omega_{2}\left(g\left(\left|x_{k_{n}}^{\prime}(t)\right|\right)\right) d t \\
& \leq \frac{2 h(\Lambda \Delta)}{\Lambda} \int_{\mathcal{M}_{k_{n}}^{1}} \omega_{2}(s) d s+\frac{2 \omega_{2}(\Lambda \Delta)}{\Lambda} \int_{\mathcal{M}_{k_{n}}^{2}} h(s) d s,
\end{aligned}
$$

where $\mathcal{M}_{k_{n}}^{i} \subset[0, \Lambda T], \mu\left(\mathcal{M}_{k_{n}}^{i}\right) \leq \Lambda \sum_{j \in \mathbb{J}}\left(b_{j}-a_{j}\right)$ for $n \in \mathbb{N}$ and $i=1,2$.

The situation is more complicated for $j \in \mathbb{L}_{k_{n}}$. Passing to a subsequence if necessary, one of the following subcases of Case 1 occurs (see Lemma 5), namelym, Case 1.1 where $\left|x_{k_{n}}(T)\right|>\Lambda$ for $n \in \mathbb{N}$ and Case 1.2 where $\left|x_{k_{n}}(T)\right| \leq \Lambda$ and $c \leq \xi_{k_{n}} \leq T-c$ for $n \in \mathbb{N}$ with a positive constant $c$.

Case 1.1. Let $\left|x_{k_{n}}(T)\right|>\Lambda$ for $n \in \mathbb{N}$. Since $\xi_{k_{n}}>\eta_{k_{n}}$, we see that $\left|x_{k_{n}}\right|$ is decreasing on $\left[\xi_{k_{n}}, T\right]$ and then $\left|x_{k_{n}}(t)\right|>\Lambda$ for $t \in\left[\xi_{k_{n}}, T\right]$. Let $j \in \mathbb{L}_{k_{n}}$. If 
a) $a_{j}<\xi_{k_{n}}<b_{j}$, then $($ see $(2.37))$

$$
\begin{aligned}
& \int_{a_{j}}^{b_{j}} h\left(\left|x_{k_{n}}(t)\right|\right) \omega_{2}\left(g\left(\left|x_{k_{n}}^{\prime}(t)\right|\right)\right) d t \\
& \quad \leq h(\Lambda \Delta) \int_{a_{j}}^{\xi_{k_{n}}} \omega_{2}\left(\Lambda\left(\xi_{k_{n}}-t\right)\right) d t+h(\Lambda) \int_{\xi_{k_{n}}}^{b_{j}} \omega_{2}\left(\Lambda\left(t-\xi_{k_{n}}\right)\right) d t \\
& \quad=\frac{h(\Lambda \Delta)}{\Lambda} \int_{0}^{\Lambda\left(\xi_{k_{n}}-a_{j}\right)} \omega_{2}(s) d s+\frac{h(\Lambda)}{\Lambda} \int_{0}^{\Lambda\left(b_{j}-\xi_{k_{n}}\right)} \omega_{2}(s) d s ;
\end{aligned}
$$

b) $\xi_{k_{n}} \leq a_{j}$, then

$$
\begin{aligned}
\int_{a_{j}}^{b_{j}} h\left(\left|x_{k_{n}}(t)\right|\right) \omega_{2}\left(g\left(\left|x_{k_{n}}^{\prime}(t)\right|\right)\right) d t & \leq h(\Lambda) \int_{a_{j}}^{b_{j}} \omega_{2}\left(\Lambda\left(t-\xi_{k_{n}}\right)\right) d t \\
& =\frac{h(\Lambda)}{\Lambda} \int_{\Lambda\left(a_{j}-\xi_{k_{n}}\right)}^{\Lambda\left(b_{j}-\xi_{k_{n}}\right)} \omega_{2}(s) d s
\end{aligned}
$$

It follows that

$$
\sum_{j \in \mathbb{L}_{k_{n}}} \int_{a_{j}}^{b_{j}} h\left(\left|x_{k_{n}}(t)\right|\right) \omega_{2}\left(g\left(\left|x_{k_{n}}^{\prime}(t)\right|\right)\right) d t \leq \frac{\max \{h(\Lambda), h(\Lambda \Delta)\}}{\Lambda} \int_{\mathcal{M}_{k_{n}}^{3}} \omega_{2}(s) d s
$$

where $\mathcal{M}_{k_{n}}^{3} \subset[0, \Lambda T]$ and $\mu\left(\mathcal{M}_{k_{n}}^{3}\right) \leq \Lambda \sum_{j \in \mathbb{J}}\left(b_{j}-a_{j}\right)$ for $n \in \mathbb{N}$.

Case 1.2. Let $\left|x_{k_{n}}(T)\right| \leq \Lambda$ for $n \in \mathbb{N}$ and

$$
c \leq \xi_{k_{n}} \leq T-c \quad \text { for } n \in \mathbb{N} .
$$

Since

and

$$
T-t \geq T-\frac{T+\xi_{k_{n}}}{2} \geq \frac{T-\xi_{k_{n}}}{2} \geq \frac{c}{2}, \quad t \in\left[\xi_{k_{n}},\left(\xi_{k_{n}}+T\right) / 2\right],
$$

$$
t-\xi_{k_{n}} \geq \frac{T+\xi_{k_{n}}}{2}-\xi_{k_{n}} \geq \frac{T-\xi_{k_{n}}}{2} \geq \frac{c}{2}, \quad t \in\left[\left(\xi_{k_{n}}+T\right) / 2, T\right],
$$

then from (2.36) and (2.37) we have

$$
g\left(\left|x_{k_{n}}^{\prime}(t)\right|\right) \geq \begin{cases}\Lambda\left|t-\xi_{k_{n}}\right| & \text { for } t \in\left[\left(\eta_{k_{n}}+\xi_{k_{n}}\right) / 2,\left(\xi_{k_{n}}+T\right) / 2\right], \\ \Lambda c / 2 & \text { for } t \in\left[\left(\xi_{k_{n}}+T\right) / 2, T\right]\end{cases}
$$

and

$$
\left|x_{k_{n}}(t)\right| \geq \begin{cases}\Lambda \Delta & \text { for } t \in\left[\left(\eta_{k_{n}}+\xi_{k_{n}}\right) / 2, \xi_{k_{n}}\right] \\ \Lambda c / 2 & \text { for } t \in\left[\xi_{k_{n}},\left(\xi_{k_{n}}+T\right) / 2\right] \\ \Lambda(T-t) & \text { for } t \in\left[\left(\xi_{k_{n}}+T\right) / 2, T\right]\end{cases}
$$

Let $j \in \mathbb{L}_{k_{n}}$ and assume that $b_{j}-a_{j} \leq c / 4$. Then $\left(T-\xi_{k_{n}}\right) / 2 \geq c / 2>c / 4 \geq b_{j}-a_{j}$ for $n \in \mathbb{N}$. If 
(a) $a_{j} \leq \xi_{k_{n}}<b_{j}<\left(\xi_{k_{n}}+T\right) / 2$, , then (see (2.42))

$$
\begin{aligned}
\int_{a_{j}}^{b_{j}} h\left(\left|x_{k_{n}}(t)\right|\right) \omega_{2}\left(g\left(\left|x_{k_{n}}^{\prime}(t)\right|\right)\right) d t \\
\quad \leq h(\Lambda \Delta) \int_{a_{j}}^{\xi_{k_{n}}} \omega_{2}\left(\Lambda\left(\xi_{k_{n}}-t\right)\right) d t+h(\Lambda c / 2) \int_{\xi_{k_{n}}}^{b_{j}} \omega_{2}\left(\Lambda\left(t-\xi_{k_{n}}\right)\right) d t \\
\quad=\frac{h(\Lambda \Delta)}{\Lambda} \int_{0}^{\Lambda\left(\xi_{k_{n}}-a_{j}\right)} \omega_{2}(s) d s+\frac{h(\Lambda c / 2)}{\Lambda} \int_{0}^{\Lambda\left(b_{j}-\xi_{k_{n}}\right)} \omega_{2}(s) d s
\end{aligned}
$$

(b) $\xi_{k_{n}}<a_{j}<b_{j} \leq\left(\xi_{k_{n}}+T\right) / 2$, then

$$
\begin{aligned}
\int_{a_{j}}^{b_{j}} h\left(\left|x_{k_{n}}(t)\right|\right) \omega_{2}\left(g\left(\left|x_{k_{n}}^{\prime}(t)\right|\right)\right) d t & \leq h(\Lambda c / 2) \int_{a_{j}}^{b_{j}} \omega_{2}\left(\Lambda\left(t-\xi_{k_{n}}\right)\right) d t \\
& =\frac{h(\Lambda c / 2)}{\Lambda} \int_{\Lambda\left(a_{j}-\xi_{k_{n}}\right)}^{\Lambda\left(b_{j}-\xi_{k_{n}}\right)} \omega_{2}(s) d s
\end{aligned}
$$

(c) $\xi_{k_{n}}<a_{j}<\left(\xi_{k_{n}}+T\right) / 2<b_{j}$, then (see (2.42) and (2.43))

$$
\begin{aligned}
\int_{a_{j}}^{b_{j}} h\left(\left|x_{k_{n}}(t)\right|\right) \omega_{2}\left(g\left(\left|x_{k_{n}}^{\prime}(t)\right|\right)\right) d t & \\
\quad \leq & h(\Lambda c / 2) \int_{a_{j}}^{\left(\xi_{k_{n}}+T\right) / 2} \omega_{2}\left(\Lambda\left(t-\xi_{k_{n}}\right)\right) d t \\
& \quad+\omega_{2}(\Lambda c / 2) \int_{\left(\xi_{k_{n}}+T\right) / 2}^{b_{j}} h(\Lambda(T-t)) d t \\
= & \frac{h(\Lambda c / 2)}{\Lambda} \int_{\Lambda\left(a_{j}-\xi_{k_{n}}\right)}^{\Lambda\left(T-\xi_{k_{n}}\right) / 2} \omega_{2}(s) d s+\frac{\omega_{2}(\Lambda c / 2)}{\Lambda} \int_{\Lambda\left(b_{j}-\xi_{k_{n}}\right)}^{\Lambda\left(T-\xi_{k_{n}}\right) / 2} h(s) d s ;
\end{aligned}
$$

(d) $\left(\xi_{k_{n}}+T\right) / 2 \leq a_{j}$, then

$$
\begin{aligned}
\int_{a_{j}}^{b_{j}} h\left(\left|x_{k_{n}}(t)\right|\right) \omega_{2}\left(g\left(\left|x_{k_{n}}^{\prime}(t)\right|\right)\right) d t & \leq \omega_{2}(\Lambda c / 2) \int_{a_{j}}^{b_{j}} h(\Lambda(T-t)) d t \\
& =\frac{\omega_{2}(\Lambda c / 2)}{\Lambda} \int_{\Lambda\left(T-b_{j}\right)}^{\Lambda\left(T-a_{j}\right)} h(s) d s
\end{aligned}
$$

"We see that then $a_{j} \geq b_{j}-\Delta>\xi_{k_{n}}-\Delta \geq \xi_{k_{n}}-\left(\xi_{k_{n}}-\eta_{k_{n}}\right) / 2=\left(\xi_{k_{n}}+\eta_{k_{n}}\right) / 2$. 
It follows that

$$
\begin{aligned}
\sum_{j \in \mathbb{L}_{k_{n}}} \int_{a_{j}}^{b_{j}} h\left(\left|x_{k_{n}}(t)\right|\right) & \omega_{2}\left(g\left(\left|x_{k_{n}}^{\prime}(t)\right|\right)\right) d t \\
\leq & \frac{2 h(\Lambda c / 2)}{\Lambda} \int_{\mathcal{N}_{k_{n}}^{1}} \omega_{2}(s) d s+\frac{2 \omega_{2}(\Lambda c / 2)}{\Lambda} \int_{\mathcal{N}_{k_{n}}^{2}} h(s) d s
\end{aligned}
$$

where $\mathcal{N}_{k_{n}}^{i} \subset[0, \Lambda T], \mu\left(\mathcal{N}_{k_{n}}^{i}\right) \leq \Lambda \sum_{j \in \mathbb{J}}\left(b_{j}-a_{j}\right)$ for $n \in \mathbb{N}$ and $i=1,2$.

Summarizing, since $h, \omega_{2} \in L_{1}([0, \Lambda T])$, from (2.39), (2.40) and (2.44) we deduce that $\left\{h\left(\left|x_{k_{n}}(t)\right|\right) \omega_{2}\left(g\left(\left|x_{k_{n}}^{\prime}(t)\right|\right)\right)\right\}$ is UI on $J$.

Case 2. Let $\eta_{k_{n}}>\xi_{k_{n}}$ for $n \in \mathbb{N}$. Define (for $n \in \mathbb{N}$ and $t \in J$ )

$$
\eta_{k_{n}}^{*}=T-\eta_{k_{n}}, \quad \xi_{k_{n}}^{*}=T-\xi_{k_{n}}
$$

and

$$
y_{k_{n}}(t)=x_{k_{n}}(T-t) .
$$

Then $\eta_{k_{n}}^{*}<\xi_{k_{n}}^{*}, \eta_{k_{n}}^{*}$ and $\xi_{k_{n}}^{*}$ is a zero of $y_{k_{n}}$ and $y_{k_{n}}^{\prime}$, respectively. Next

$$
\left|\eta_{k_{n}}^{*}-\xi_{k_{n}}^{*}\right|=\left|\left(T-\eta_{k_{n}}\right)-\left(T-\xi_{k_{n}}\right)\right|=\left|\xi_{k_{n}}-\eta_{k_{n}}\right| \geq \Delta / 2, \quad n \in \mathbb{N}
$$

where $\Delta$ is given by Lemma 4. In addition, by Lemma 1 (see (2.4) and (2.5)) we have (for $n \in \mathbb{N}$ )

$$
g\left(\left|y_{k_{n}}^{\prime}(t)\right|\right)=g\left(\left|x_{k_{n}}^{\prime}(T-t)\right|\right) \geq \Lambda\left|(T-t)-\xi_{k_{n}}\right|=\Lambda\left|t-\xi_{k_{n}}^{*}\right|, \quad t \in[0, T]
$$

and

$$
\begin{aligned}
\left|y_{k_{n}}(t)\right| & =\left|x_{k_{n}}(T-t)\right| \\
& \geq \begin{cases}\Lambda\left|(T-t)-\eta_{k_{n}}\right|=\Lambda\left|\eta_{k_{n}}^{*}-t\right|, & \xi_{k_{n}} \leq T-t \leq T\left(\Longleftrightarrow t \in\left[0, \xi_{k_{n}}^{*}\right]\right), \\
\Lambda(T-t), & 0 \leq T-t<\xi_{k_{n}}\left(\Longleftrightarrow t \in\left(\xi_{k_{n}}^{*}, T\right]\right) .\end{cases}
\end{aligned}
$$

By Case 1, the sequence

$$
\left\{h\left(\left|y_{k_{n}}(t)\right|\right) \omega_{2}\left(g\left(\left|y_{k_{n}}^{\prime}(t)\right|\right)\right)\right\}
$$

is UI on $J$. Since

$$
\sum_{j \in \mathbb{J}} \int_{a_{j}}^{b_{j}} h\left(\left|x_{k_{n}}(t)\right|\right) \omega_{2}\left(g\left(\left|x_{k_{n}}^{\prime}(t)\right|\right)\right) d t=\sum_{j \in \mathbb{J}} \int_{T-b_{j}}^{T-a_{j}} h\left(\left|y_{k_{n}}(s)\right|\right) \omega_{2}\left(g\left(\left|y_{k_{n}}^{\prime}(s)\right|\right)\right) d s
$$

and

$$
\sum_{j \in \mathbb{J}}\left(b_{j}-a_{j}\right)=\sum_{j \in \mathbb{J}}\left[\left(T-a_{j}\right)-\left(T-b_{j}\right)\right],
$$

we see that the sequence $\left\{h\left(\left|x_{k_{n}}(t)\right|\right) \omega_{2}\left(g\left(\left|x_{k_{n}}^{\prime}(t)\right|\right)\right)\right\}$ is UI on $J$. 


\section{EXISTENCE RESULTS AND AN EXAMPLE}

Theorem. Let assumptions $\left(H_{1}\right)-\left(H_{3}\right)$ be satisfied. Then BVP (1.1), (1.2) has a solution.

Proof. By Lemma 3, for each $n \in \mathbb{N}$, there exists a solution $x_{n}$ of BVP (2.3), (1.2) with $\lambda=1$ in (2.3). Let $\eta_{n} \in[0, T)$ and $\xi_{n} \in(0, T)$ be the unique zero of $x_{n}$ on $[0, T)$ and $x_{n}^{\prime}$, respectively. Without loss of generality, we can assume that $\xi_{n}<\eta_{n}$ (then $\eta_{n}>0$ ) for $n \in \mathbb{N}$. Now Lemma 1 shows that, for each $n \in \mathbb{N}$, we have

$$
g\left(\left|x_{n}^{\prime}(t)\right|\right) \geq \Lambda\left|t-\xi_{n}\right| \quad \text { for } \quad t \in J
$$

and

$$
\left|x_{n}(t)\right| \geq \begin{cases}\Lambda t & \text { for } t \in\left[0, \xi_{n}\right] \\ \Lambda\left|t-\eta_{n}\right| & \text { for } t \in\left(\xi_{n}, T\right]\end{cases}
$$

where $\Lambda$ is given by (2.31). Applying Lemmas 2 and 4, there exist positive constants $P$ and $\Delta$ such that the inequalities (2.32) are satisfied. In addition, without loss of generality we can also assume that the sequence $\left\{f_{n}\left(t, x_{n}(t), x_{n}^{\prime}(t)\right)\right\}$ is UI on $J$ by Lemma 6, which guarantees that $\left\{g\left(x_{n}^{\prime}(t)\right)\right\}$ is equicontinuous on $J$, and then from

$$
\left|x_{n}^{\prime}\left(t_{2}\right)-x_{n}^{\prime}\left(t_{1}\right)\right|=\left|g^{-1}\left(g\left(x_{n}^{\prime}\left(t_{2}\right)\right)\right)-g^{-1}\left(g\left(x_{n}^{\prime}\left(t_{1}\right)\right)\right)\right|, \quad t_{1}, t_{2} \in J,
$$

the uniform continuity of $g^{-1}$ on $[-g(P), g(P)]$ and the fact, that $g^{-1}$ is increasing, we deduce that $\left\{x_{n}^{\prime}(t)\right\}$ is equicontinuous on $J$ as well. On account of the Arzelà-Ascoli theorem and the Bolzano-Weierstrass theorem, passing to subsequences if necessary, we can assume that $\left\{x_{n}\right\}$ is convergent in $C^{1}(J)$ and $\left\{\eta_{n}\right\},\left\{\xi_{n}\right\}$ are convergent in $\mathbb{R}$. Let $\lim _{n \rightarrow \infty} x_{n}=x, \lim _{n \rightarrow \infty} \eta_{n}=\eta$ and $\lim _{n \rightarrow \infty} \xi_{n}=\xi$. Then $x \in C^{1}(J), x$ satisfied (1.2) and $\Delta \leq|\xi-\eta|$. Letting $n \rightarrow \infty$ in (3.1) and (3.2), we get

$$
\left|x^{\prime}(t)\right| \geq g^{-1}(\Lambda|t-\xi|) \quad \text { for } \quad t \in J
$$

and

$$
|x(t)| \geq \begin{cases}\Lambda t & \text { for } t \in[0, \xi], \\ \Lambda|t-\eta| & \text { for } t \in(\xi, T] .\end{cases}
$$

From the construction of the functions $f_{n} \in \operatorname{Car}\left(J \times \mathbb{R}^{2}\right)$ it follows the existence of a $\mathcal{U} \subset J, \mu(\mathcal{U})=0$, such that $f_{n}(t, \cdot, \cdot)$ is continuous on $\mathbb{R}^{2}$ for each $t \in J \backslash \mathcal{U}$ and $n \in \mathbb{N}$. Then (see (3.3) and (3.4))

$$
\lim _{n \rightarrow \infty} f_{n}\left(t, x_{n}(t), x_{n}^{\prime}(t)\right)=f\left(t, x(t), x^{\prime}(t)\right), \quad t \in J \backslash(\mathcal{U} \cup\{0, \eta, \xi\}) .
$$

Therefore $f\left(t, x(t), x^{\prime}(t)\right) \in L_{1}(J)$ and

$$
\lim _{n \rightarrow \infty} \int_{0}^{t} f_{n}\left(s, x_{n}(s), x_{n}^{\prime}(s)\right) d s=\int_{0}^{t} f\left(s, x(s), x^{\prime}(s)\right) d s
$$


for $t \in J$ by Vitali's convergence theorem. Now, taking the limit as $n \rightarrow \infty$ in the equalities

we obtain

$$
g\left(x_{n}^{\prime}(t)\right)=g\left(x_{n}^{\prime}(0)\right)+\int_{0}^{t} f_{n}\left(s, x_{n}(s), x_{n}^{\prime}(s)\right) d s, \quad t \in J, \quad n \in \mathbb{N},
$$

$$
g\left(x^{\prime}(t)\right)=g\left(x^{\prime}(0)\right)+\int_{0}^{t} f\left(s, x(s), x^{\prime}(s)\right) d s, \quad t \in J .
$$

Hence $g\left(x^{\prime}\right) \in A C(J)$ and $x$ is a solution of (1.1). We have proved that $x$ is a solution of BVP (1.1), (1.2).

If $f \in C^{0}\left(J \times \mathbb{R}_{0}^{2}\right)$, then the theorem given above immediately yields

Corollary. Let $f \in C^{0}\left(J \times \mathbb{R}_{0}^{2}\right)$ and assumptions $\left(H_{1}\right)-\left(H_{3}\right)$ be satisfied. Then there exists a solution $x$ of $B V P(1.1),(1.2)$. If $\eta \in[0, T)$ and $\xi \in(0, T)$ denotes the (unique) zero of $x$ on $[0, T)$ and $x^{\prime}$, respectively, then $g\left(x^{\prime}\right) \in C^{1}(J \backslash \mathcal{U})$ and (1.1) is satisfied for $t \in J \backslash \mathcal{U}$ where $\mathcal{U}=\{0, \xi, T\}$ if $\eta=0$ and $\mathcal{U}=\{\eta, \xi\}$ if $\eta>0$.

Example. Let $p \in(0, \infty), q_{i} \in L_{\infty}(J)$ be nonnegative, $\alpha_{j} \in(0,1)$ and $\gamma_{j} \in(0, p)$ $(i=1,2,3$ and $j=1,2)$. Consider the differential equation

$$
\begin{aligned}
\left(\left|x^{\prime}(t)\right|^{p} \operatorname{sign} x^{\prime}(t)\right)^{\prime}=\arctan (1+ & |x(t)|) \\
& +\frac{q_{1}(t)}{|x(t)|^{\alpha_{1}}}+q_{2}(t)\left|x^{\prime}(t)\right|^{\gamma_{1}}+\frac{q_{3}(t)}{|x(t)|^{\alpha_{2}}\left|x^{\prime}(t)\right|^{\gamma_{2}}} .
\end{aligned}
$$

Problem (3.5), (1.2) is the special case of BVP (1.1), (1.2) with $g(u)=|u|^{p}$ sign $u$ and

$$
f(t, u, v)=\arctan (1+|u|)+\frac{q_{1}(t)}{|u|^{\alpha_{1}}}+q_{2}(t)|v|^{\gamma_{1}}+\frac{q_{3}(t)}{|u|^{\alpha_{2}}|v|^{\gamma_{2}}} .
$$

Assumptions $\left(H_{1}\right)-\left(H_{3}\right)$ are satisfied with $a=\arctan 1, \omega_{1}(u)=2+u^{\gamma_{1} / p}, \omega_{2}(u)=$ $1 / u^{\gamma_{2} / p}$, and

$$
h(u)=K\left(1+1 / u^{\alpha_{1}}+1 / u^{\alpha_{2}}\right)
$$

where $K=\max \left\{\frac{\pi}{2},\left\|q_{j}\right\|_{\infty} ; j=1,2,3\right\}$. Applying the theorem, we show that there exists a solution of BVP (3.5), (1.2).

\section{REFERENCES}

[1] Agarwal, R. P., O’Regan, D., Rachůnková, I., StanĚK, S.: Two-point higher-order boundary value problems with singularities in phase variables, Comput. Math. Appl., 46 (2003), 1799-1826.

[2] Agarwal, R. P., O'Regan, D., Staněk, S.: Solvability of singular boundary value problems with given maximal values for solutions, (to appear).

[3] BartLe, R.G.: A modern theory of integration, RI: American Mathematical Society (AMS), Providence, 2001.

[4] BRYkalov, S.A.: Problems for ordinary differential equations with monotone boundary conditions, Differentsial'nye Uravneniya, 32 (1996), 1322-1330 (in Russian).

[5] Brykalov, S.A.: Solvability of problems with monotone boundary conditions, Differentsial'nye Uravneniya, 29 (1993), 744-750 (in Russian); English transl. in Differential Equations, 29 (1993), 633-639. 
[6] Granas, A., Guenther, R. B., Lee, J. W.: Nonlinear boundary value problems for ordinary differential equations, Diss. Math., 244, (1985), 128 p.

[7] Granas, A., Guenther, R.B., Lee, J.W.: Some general existence principles in the Carathéodory theory of nonlinear differential systems, J. Math. Pures Appl., IX. Ser., 70 (1991), No. 2, 153-196.

[8] KIgURADze, I.T.: Boundary value problems for systems of ordinary differential equations, Itogi Nauki Tekh., Ser. Sovrem. Probl. Mat., 30 (1987), 3-103 (in Russian).

[9] RachŮNкоvá, I., StaněK, S.: A singular boundary value problem for odd-order differential equations, J. Math. Anal. Appl., 291 (2004), No. 2, 741-756.

[10] RachŮnková, I., StaněK, S.: Sign-changing solutions of singular Dirichlet boundary value problems, Arch. Inequal. Appl., 1 (2003), 11-30.

[11] STANĚK, S.: Functional boundary value problems for second order functional differential equations of the neutral type, Glas. Mat., III. Ser., 36 (2001), 73-84.

[12] StanĚK, S.: Two functional boundary value problems with singularities in phase variables, Nonlinear Oscil., 5 (2002), 387-415.

[13] STANĚK, S.: A nonlocal singular boundary value problem for second-order differential equations, Miskolc Math. Notes, 5 (2004), No. 1, 91-104.

[14] STANĚK, S.: On a nonlocal singular boundary value problem with singularities in phase variables, Mathematical and Computer Modelling (in press).

\section{Author's Address}

\section{Oto Přibyl:}

Brno University of Technology, Faculty of Civil Engineering, Institute of Mathematics, Veveří 331/95, 60200 Brno, Czech Republic

E-mail address: pribyl.o@fce.vutbr.cz 